\title{
Self-Constructing Compact Parametric Macromodels for Efficient Electronic Design
}

\author{
Francesco Ferranti, Krishnan Chemmangat, Tom Dhaene, Luc Knockaert \\ Department of Information Technology (INTEC), Ghent University-IMINDS, \\ Gaston Crommenlaan 8 Bus 201, 9050 Ghent, Belgium \\ Email: \{francesco.ferranti, krishnan.cmc, tom.dhaene, luc.knockaert\}@ugent.be
}

\begin{abstract}
We propose a modeling technique able to generate very efficient parametric macromodels in an automated way. The design space samples needed to build the parametric macromodels are chosen automatically by means of a suitable adaptive sampling algorithm. The size of the parametric macromodels does not increase with the number of design parameters, which leads to compact models. Stability and passivity are preserved over the design space of interest. Pertinent numerical results validate the proposed approach.
\end{abstract}

\section{INTRODUCTION}

During a typical design process of modern high-speed and high-performance systems many electromagnetic (EM) simulations must be performed for various combinations of the design variables (e.g., layout features) in order to perform design optimization, design space exploration and variability analysis. In this perspective, compact parametric macromodels are well suited and preferred to efficiently and accurately perform these design activities, instead of calculating multiple computationally expensive EM simulations on-the-fly. The accuracy, compactness and preservation of system properties (e.g. stability and passivity) are fundamental and desired features of such parametric macromodels.

Over the years, different passivity-preserving parametric macromodeling techniques have been proposed [1]-[6]. Recently, a parametric macromodeling method able to generate compact, stable and passive models over the entire design space of interest has been proposed in [6]. It is based on the use of reference macromodels and positive interpolation operators. The design space is divided into cells and each cell is modeled independently. The model order is not increasing with the number of design parameters, and this leads to compact models.

The selection of data samples in the multidimensional design space is of paramount importance for an efficient and automated construction of the parametric macromodels. Adaptive sampling methods are fundamental to automatically sample the design space with a minimum amount of properly located samples. Recently, an interesting adaptive sampling technique has been proposed in [7]. It uses a local approach and works on local hyper-rectangular regions of the design space. This creates the possibility of a tree-based implementation of the sampling algorithm, while making it portable to parallel computing platforms.

This paper proposes a modeling approach to generate very efficient parametric macromodels in an automated way. A sampling algorithm similar to [7] and the parametric macromodeling approach of [6] are properly combined to provide a powerful tool to speed-up design activities of modern highspeed and high-performance systems. Pertinent numerical results validate the proposed methodology.

\section{A. Parametric macromodeling technique}

A parametric macromodel in a state-space form:

$$
\mathbf{R}(s, \mathbf{g})=\mathbf{C}(\mathbf{g})(s \mathbf{I}-\mathbf{A}(\mathbf{g}))^{-1} \mathbf{B}(\mathbf{g})+\mathbf{D}(\mathbf{g})
$$

or equivalently in a pole-residue form:

$$
\mathbf{R}(s, \mathbf{g})=\mathbf{C}_{0}(\mathbf{g})+\sum_{n=1}^{N(\mathbf{g})} \frac{\mathbf{C}_{n}(\mathbf{g})}{s-p_{n}(\mathbf{g})}
$$

is computed by the parametric macromodeling method [6] to accurately model a set of multivariate data samples $\left\{(s, \mathbf{g})_{k}, \mathbf{H}(s, \mathbf{g})_{k}\right\}_{k=1}^{K_{t o t}}$, which depend on the complex frequency $s=j \omega$ and $M$ design variables $\mathbf{g}=\left(\mathrm{g}^{(m)}\right)_{m=1}^{M}$, such as layout features or substrate parameters. The design space $\mathcal{D}(\mathbf{g})$ contains all design parameters $\mathbf{g}=\left(\mathrm{g}^{(m)}\right)_{m=1}^{M}$.

As a first step, the design space is divided into cells. We indicate a cell region of the design space as $\Omega_{i}, i=1, \ldots, P$ and the corresponding vertices as $\mathbf{g}_{k}^{\Omega_{i}}, k=1, \ldots, Q$. For each cell a reference macromodel is computed in the form:

$$
\mathbf{R}\left(s, \mathbf{g}_{k}^{\Omega_{i}}\right)=\mathbf{C}_{0}\left(\mathbf{g}_{k}^{\Omega_{i}}\right)+\sum_{n=1}^{N\left(\Omega_{i}\right)} \frac{\mathbf{C}_{n}\left(\mathbf{g}_{k}^{\Omega_{i}}\right)}{s \alpha\left(\mathbf{g}_{k}^{\Omega_{i}}\right)-p_{r e f, n}\left(\Omega_{i}\right)}
$$

using the data samples $\mathbf{H}\left(s, \mathbf{g}_{k}^{\Omega_{i}}\right)$, the Vector Fitting (VF) method [8], optimization steps, linear least-squares solutions and passivity enforcement techniques [6]. Each design space cell is described by its reference poles, vertex frequency scaling coefficients, residues and direct terms $p_{\text {ref,n }}\left(\Omega_{i}\right)$, $\alpha\left(\mathbf{g}_{k}^{\Omega_{i}}\right), \mathbf{C}_{n}\left(\mathbf{g}_{k}^{\Omega_{i}}\right), \mathbf{C}_{0}\left(\mathbf{g}_{k}^{\Omega_{i}}\right), \quad k=1, \ldots, Q$. The reference poles are computed at one cell vertex (reference point) for which $\alpha=1$.

The next step is to build a parametric macromodel $\mathbf{R}(s, \mathbf{g})$ using suitable interpolation schemes that are applied to the vertex frequency scaling coefficients, residues and direct terms for each design space cell. To describe the parametric macromodeling algorithm more clearly, we consider a specific cell $\widehat{\Omega}$ composed of 4 vertices in a 2-D design space $\mathbf{g}=\left(\mathrm{g}^{(1)}, \mathrm{g}^{(2)}\right)$, as shown in Fig. 1. 


$$
\mathbf{R}\left(s, \mathbf{g}_{k}^{\widehat{\Omega}}\right)=\mathbf{C}_{0}\left(\mathbf{g}_{k}^{\widehat{\Omega}}\right)+\Sigma_{n=1}^{N(\widehat{\Omega})} \frac{\mathbf{C}_{n}\left(\mathbf{g}_{k}^{\widehat{\Omega}}\right)}{s \alpha\left(\mathbf{g}_{k}^{\hat{\Omega}}\right)-\mathbf{p}_{r e f, n}(\widehat{\Omega})}
$$

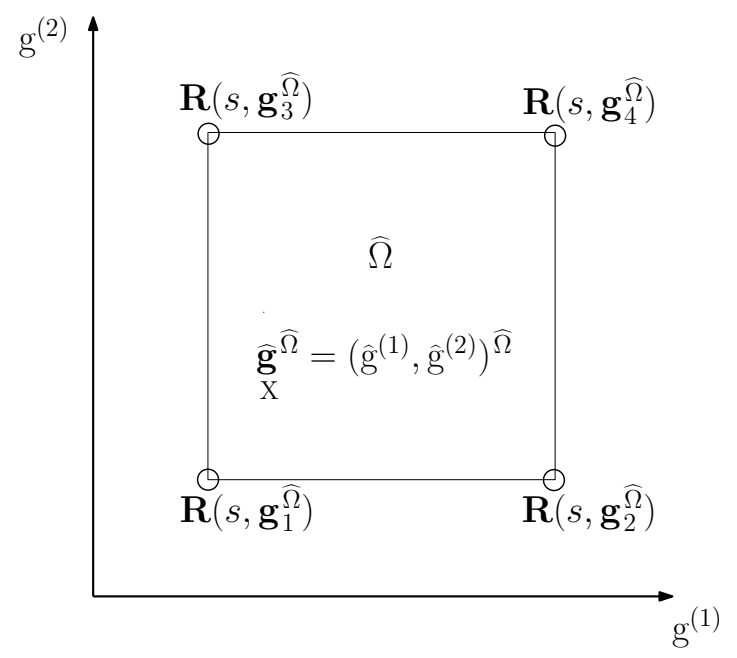

Fig. 1. 2-D design space cell $\widehat{\Omega}$.

If the response of the system under modeling needs to be computed at a specific point of the design space cell $\widehat{\mathbf{g}}^{\widehat{\Omega}}=\left(\widehat{\mathrm{g}}^{(1)}, \widehat{\mathrm{g}}^{(2)}\right)^{\widehat{\Omega}}$, the frequency scaling coefficients $\alpha\left(\mathbf{g}_{k}^{\widehat{\Omega}}\right)$, residues $\mathbf{C}_{n}\left(\mathbf{g}_{k}^{\widehat{\Omega}}\right)$, direct terms $\mathbf{C}_{0}\left(\mathbf{g}_{k}^{\widehat{\Omega}}\right)$ are interpolated in $\widehat{\mathbf{g}} \widehat{\Omega}$ and a rational model

$$
\mathbf{R}\left(s, \widehat{\mathbf{g}}^{\widehat{\Omega}}\right)=\mathbf{C}_{0}\left(\widehat{\mathbf{g}}^{\widehat{\Omega}}\right)+\sum_{n=1}^{N(\widehat{\Omega})} \frac{\mathbf{C}_{n}\left(\widehat{\mathbf{g}}^{\widehat{\Omega}}\right)}{s \alpha\left(\widehat{\mathbf{g}}^{\widehat{\Omega}}\right)-\mathbf{p}_{r e f, n}(\widehat{\Omega})}
$$

is obtained. Just as an example, let us use the piecewise multilinear method [9] for the interpolation, therefore we have for $k=1, \ldots, 4$ :

$$
\begin{aligned}
& \alpha\left(\widehat{\mathbf{g}}^{\widehat{\Omega}}\right)= \\
& =\sum_{k_{1}=1}^{2} \sum_{k_{2}=1}^{2} \alpha\left(\left(\mathrm{g}_{k_{1}}^{(1)}, \mathrm{g}_{k_{2}}^{(2)}\right)^{\widehat{\Omega}}\right) \ell_{k_{1}}\left(\mathrm{~g}^{(1)}\right) \ell_{k_{2}}\left(\mathrm{~g}^{(2)}\right) \\
& \mathbf{C}_{n}\left(\widehat{\mathbf{g}}^{\widehat{\Omega}}\right)= \\
& =\sum_{k_{1}=1}^{2} \sum_{k_{2}=1}^{2} \mathbf{C}_{n}\left(\left(\mathrm{~g}_{k_{1}}^{(1)}, \mathrm{g}_{k_{2}}^{(2)}\right)^{\widehat{\Omega}}\right) \ell_{k_{1}}\left(\mathrm{~g}^{(1)}\right) \ell_{k_{2}}\left(\mathrm{~g}^{(2)}\right) \\
& \mathbf{C}_{0}\left(\widehat{\mathbf{g}}^{\widehat{\Omega}}\right)= \\
& =\sum_{k_{1}=1}^{2} \sum_{k_{2}=1}^{2} \mathbf{C}_{0}\left(\left(\mathrm{~g}_{k_{1}}^{(1)}, \mathrm{g}_{k_{2}}^{(2)}\right)^{\widehat{\Omega}}\right) \ell_{k_{1}}\left(\mathrm{~g}^{(1)}\right) \ell_{k_{2}}\left(\mathrm{~g}^{(2)}\right)
\end{aligned}
$$

where each interpolation kernel function $\ell_{k_{j}}\left(\mathrm{~g}^{(j)}\right), j=1, \ldots, 2$ is selected as in piecewise linear interpolation. The extension of the described procedure to the general multidimensional case is straightforward.

\section{B. Adaptive sampling algorithm}

An adaptive sampling algorithm must be able to select the optimum number of samples such that accurate parametric models can be generated efficiently. Recently, an interesting adaptive sampling technique has been proposed in [7]. It uses a local approach and works on local hyper-rectangular regions of the design space. This creates the possibility of a tree-based implementation of the sampling algorithm, while making it portable to parallel computing platforms.

We propose a modification of the algorithm presented in [7]. The new algorithm divides each design space cell into two halves by selecting new samples along the edge of the most dynamic design parameter. This is a key difference with respect to the approach in [7], where each design space cell is divided at the geometric center. It allows to reduce the overall number of points needed to create the parametric models.

As an example, we consider a 2-D design space $\mathbf{g}=$ $\left(\mathrm{g}^{(1)}, \mathrm{g}^{(2)}\right)$ as shown in Fig. 2-a, where four initial design space samples are selected and corresponding sampled frequency-domain system responses $\left\{\mathbf{H}\left(s_{k}, \mathbf{g}_{i j}\right)\right\}_{k=1}^{K_{s}}, \mathbf{g}_{i j}=$ $\left(\mathrm{g}_{i}^{(1)}, \mathrm{g}_{j}^{(2)}\right), i, j=1,2$ are computed considering $k=1, \ldots, K_{s}$ and $K_{s}$ equal to the number of frequency samples. Next, rational models for the cell vertices are generated and the one with the highest number of poles provides the reference poles. Then, the reference macromodel $\mathbf{R}(s, \mathbf{g})=\mathbf{C}_{0}(\mathbf{g})+$ $\sum_{n=1}^{N(\mathbf{g})} \frac{\mathbf{C}_{n}(\mathbf{g})}{s-p_{r e f, n}(\mathbf{g})}$ for the design space cell is calculated. If the reference macromodel is accurate enough with respect to an error check at all vertices of the design space cell under study, it is interpolated and compared with frequency-domain validation data samples at the center of the cell. If sufficient accuracy is also achieved in the validation point, then the modeling of the design space cell is completed. Otherwise, the error of the reference macromodel at the $M$ closest neighbors of the reference point along each design space direction is analyzed and the design space cell is further divided into two child cells along the edge where the error is the largest, as shown in Fig. 2-c and Fig. 2-d. This procedure is repeated over the entire design space until all design space cells satisfy the error check.

The number and distribution of the selected design space samples depend on the error criteria used to check accuracy. In this work, we use the maximum absolute error (8) to assess the accuracy of the generated parametric models with a target accuracy of $-40 \mathrm{~dB}$. The maximum absolute error between the frequency-domain data samples and parametric model can be expressed as:

$$
\operatorname{Err}(\mathbf{g})=\max _{i, j, k}\left(\left|H_{i, j}\left(s_{k}, \mathbf{g}\right)-R_{i, j}\left(s_{k}, \mathbf{g}\right)\right|\right)
$$

where $i=1, \ldots, P_{\text {in }}, j=1, \ldots, P_{\text {out }}, P_{\text {in }}$ and $P_{\text {out }}$ are the number input and output ports of the system, respectively.

\section{Numerical Results}

Three coupled microstrips, as shown in Fig. 3, are modeled in this example. The length $L$ of the lines and the spacings between the lines $S_{1}, S_{2}$ are chosen as design parameters in addition to frequency. Table I shows the ranges of all parameters. The substrate is chosen with relative permittivity $\epsilon_{r}=9.6$, loss tangent $\tan \delta=0.0002$ and thickness $t=254 \mu \mathrm{m}$. 


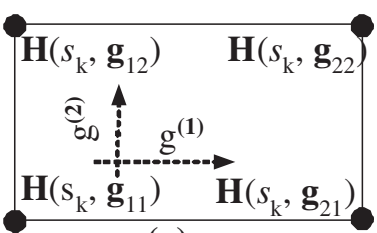

(a)

(c)

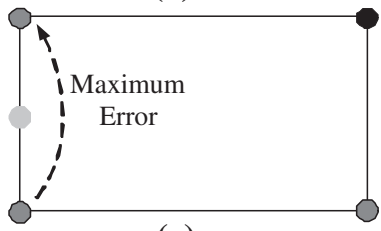

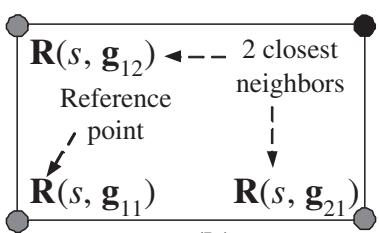

(b)

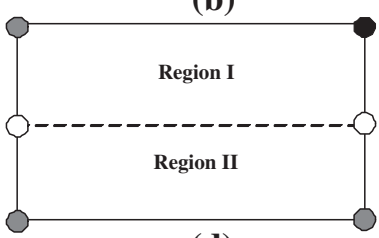

(d)
Fig. 2. Division of the design space.

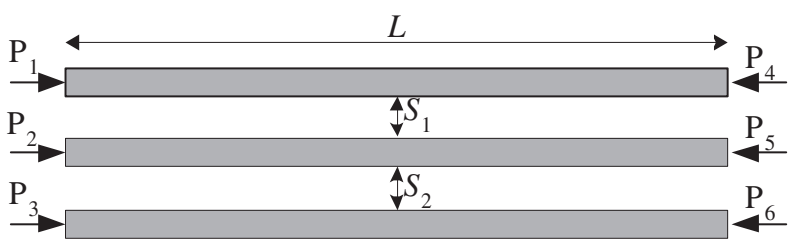

Fig. 3. Top view of the layout of three coupled microstrips.

TABLE I. DESIGN VARIABLES OF THREE COUPLED MICROSTRIPS.

\begin{tabular}{|l|c|c|}
\hline Parameter & Min & Max \\
\hline Frequency $($ freq $)$ & $0 \mathrm{GHz}$ & $10 \mathrm{GHz}$ \\
Length $(L)$ & $5 \mathrm{~mm}$ & $8 \mathrm{~mm}$ \\
Spacing $\left(S_{1}\right)$ & $0.1 \mathrm{~mm}$ & $0.15 \mathrm{~mm}$ \\
Spacing $\left(S_{2}\right)$ & $0.1 \mathrm{~mm}$ & $0.15 \mathrm{~mm}$ \\
\hline
\end{tabular}

The scattering matrix $\mathbf{S}\left(s, L, S_{1}, S_{2}\right)$ has been computed using the ADS Momentum ${ }^{1}$ software and the number of frequency samples is equal to $K_{s}=51$. Matlab R2009 $\mathrm{a}^{2}$ is used to drive the ADS Momentum simulations to generate scattering responses which are then supplied to the proposed adaptive sampling algorithm and parametric macromodeling technique [6].

The adaptive sampling algorithm selects 43 generation and 12 validation design space points to generate and validate the parametric model with an accuracy threshold of $-40 \mathrm{~dB}$. Fig. 4 shows the distribution of generation and validation samples. Fig. 5 shows the parametric behavior of the magnitude of $\mathbf{S}_{14}\left(s, L, S_{1}, S_{2}\right)$ with respect to $L$ and frequency for $S_{1}=0.12 \mathrm{~mm}, S_{2}=0.12 \mathrm{~mm}$. Similarly, the parametric behavior of the magnitude of $\mathbf{S}_{16}\left(s, L, S_{1}, S_{2}\right)$ with respect to $S_{2}$ and frequency is shown in Fig. 6 for $L=7.5 \mathrm{~mm}$, $S_{1}=0.1 \mathrm{~mm}$. Fig. 7 compares $\mathbf{S}_{14}\left(s, L, S_{1}, S_{2}\right)$ and its parametric macromodel over a design space grid of $5 \times 5 \times 5$ $\left(L, S_{1}, S_{2}\right)$ samples.

The number of poles of the parametric model is varying between 8 and 11 over the design space. The model order is not increasing with the number of design parameters, and this leads to a compact model. The proposed modeling approach is able to guarantee stability and passivity over the entire design space. We recall that a linear network described by a scattering

\footnotetext{
${ }^{1}$ Momentum EEsof EDA, Agilent Technologies, Santa Rosa, CA.

${ }^{2}$ The Mathworks, Inc., Natick, MA, USA
}

matrix $\mathbf{S}(s)$ is passive if [10]:

1) $\mathbf{S}\left(s^{*}\right)=\mathbf{S}^{*}(s)$ for all $s$, where “*” is the complex conjugate operator.

2) $\mathbf{S}(s)$ is analytic in $\Re e(s) \geq 0$.

3) $\mathbf{I}-\mathbf{S}^{T}\left(s^{*}\right) \mathbf{S}(s) \geq 0 ; \forall s: \Re e(s)>0$.

The third passivity condition is equivalent to the condition $\|\mathbf{S}(s)\|_{\infty} \leq 1$ ( $\mathbf{H}_{\infty}$ norm) [11], i.e., the largest singular value of $\mathbf{S}(s)$ does not exceed one in the right-half Laplace plane. Due to the assumption of strict stability, the third condition can be restricted to the imaginary axis of the Laplace domain.

Fig. 8 shows the $\mathbf{H}_{\infty}$ norm of the generated parametric model over a design space grid of $5 \times 5 \times 5\left(L, S_{1}, S_{2}\right)$ samples.

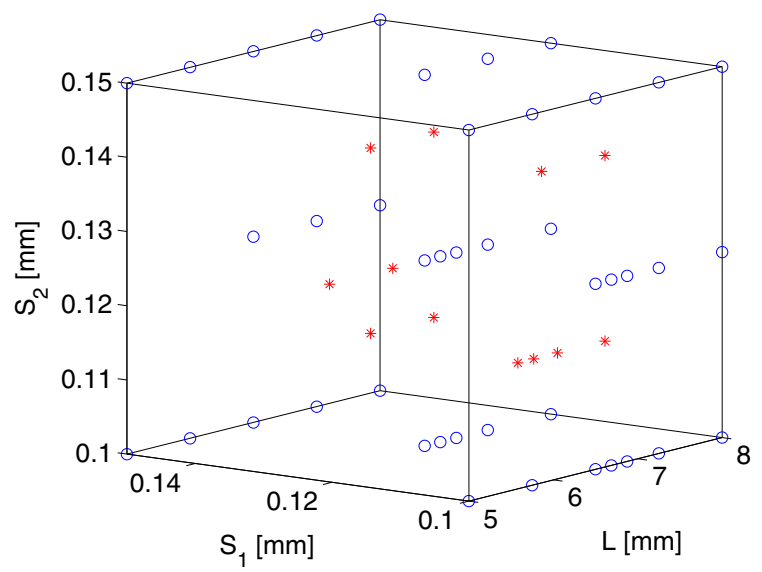

Fig. 4. Distribution of generation (o) and validation (*) design space points selected by the adaptive sampling algorithm.

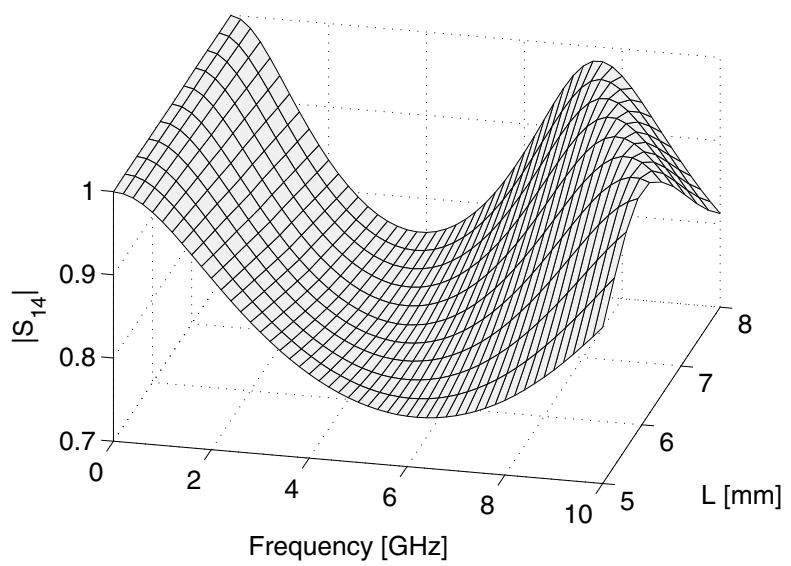

Fig. 5. Parametric behavior of the magnitude of $\mathbf{S}_{14}\left(s, L, S_{1}, S_{2}\right)$ for $S_{1}=$ $0.12 \mathrm{~mm}, S_{2}=0.12 \mathrm{~mm}$.

\section{CONCLUSION}

We have presented a modeling technique able to generate very efficient parametric macromodels in an automated way. An adaptive sampling algorithm is used to automatically select the samples needed to build the parametric models. The model order does not increase with the number of design parameters, and this results in compact models. Stability and passivity are 


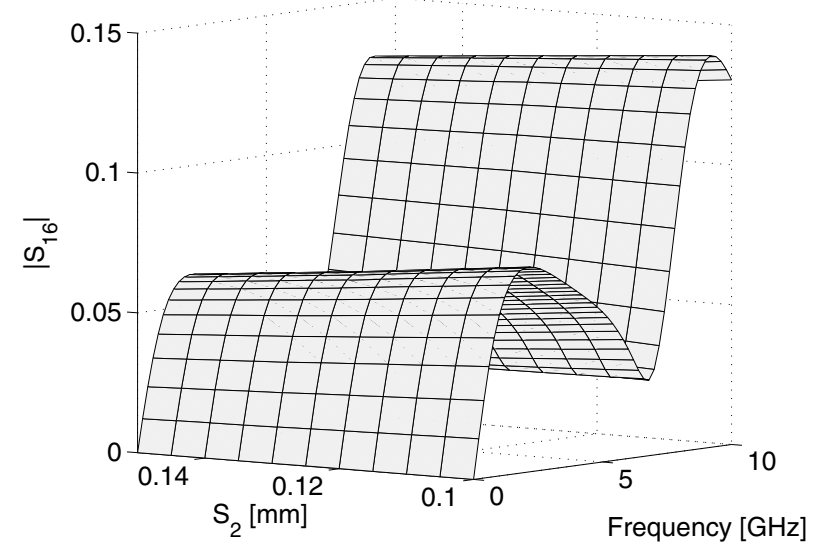

Fig. 6. Parametric behavior of the magnitude of $\mathbf{S}_{16}\left(s, L, S_{1}, S_{2}\right)$ for $L=$ $7.5 \mathrm{~mm}, S_{1}=0.1 \mathrm{~mm}$.
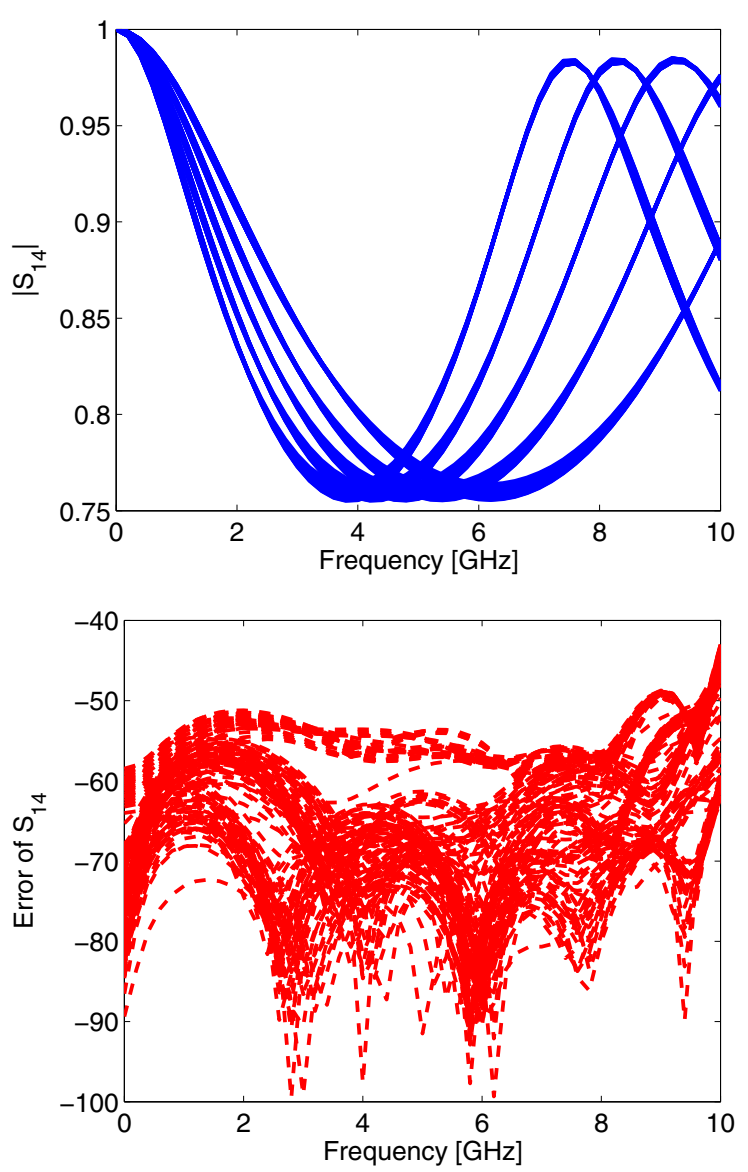

Fig. 7. Magnitude and absolute error of $\mathbf{S}_{14}\left(s, L, S_{1}, S_{2}\right)$ over a design space grid of $5 \times 5 \times 5\left(L, S_{1}, S_{2}\right)$ samples.

guaranteed over the design space of interest. The proposed approach has been validated using suitable numerical results and can be used to speed-up design activities.

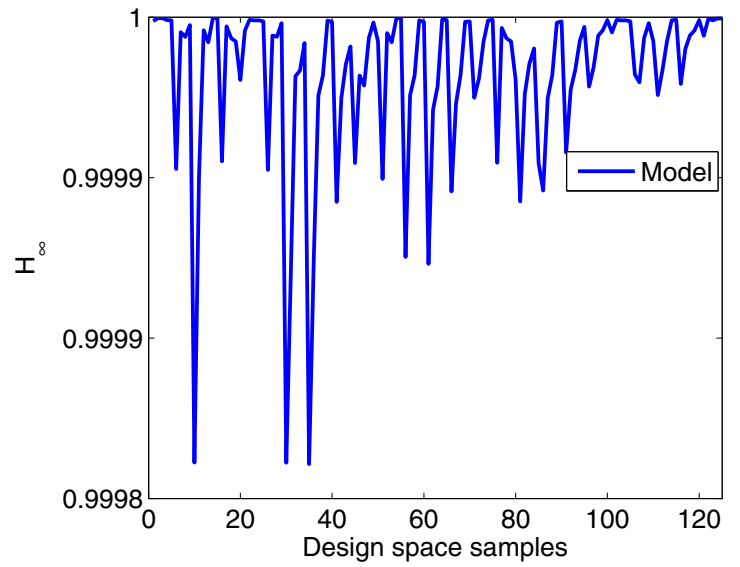

Fig. 8. $\quad \mathbf{H}_{\infty}$ norm of the parametric macromodel over a design space grid of $5 \times 5 \times 5\left(L, S_{1}, S_{2}\right)$ samples.

\section{ACKNOWLEDGMENT}

This work has been funded by the Interuniversity Attraction Poles Programme BESTCOM initiated by the Belgian Science Policy Office.

\section{REFERENCES}

[1] F. Ferranti, L. Knockaert, and T. Dhaene, "Guaranteed passive parameterized admittance-based macromodeling," IEEE Trans. Adv. Packag., vol. 33, no. 3, pp. $623-629$, Aug. 2010.

[2] _ - "Parameterized S-parameter based macromodeling with guaranteed passivity," IEEE Microw. Wireless Compon. Lett., vol. 19, no. 10, pp. 608-610, Oct. 2009.

[3] P. Triverio, M. Nakhla, and S. Grivet-Talocia, "Passive parametric macromodeling from sampled frequency data," in IEEE 14th Workshop on Signal Propagation on Interconnects (SPI), 2010, May 2010, pp. $117-120$.

[4] - "Passive parametric modeling of interconnects and packaging components from sampled impedance, admittance or scattering data," in Electronic System-Integration Technology Conference (ESTC), 2010, Sept. 2010, pp. 1-6.

[5] F. Ferranti, L. Knockaert, and T. Dhaene, "Passivity-preserving parametric macromodeling by means of scaled and shifted state-space systems," IEEE Trans. Microw. Theory Tech., vol. 59, no. 10, pp. 2394-2403, Oct. 2011.

[6] F. Ferranti, T. Dhaene, and L. Knockaert, "Compact and passive parametric macromodeling using reference macromodels and positive interpolation operators," IEEE Trans. Components, Packaging and Manufacturing Technology, vol. 2, no. 12, pp. 2080 -2088, Dec. 2012.

[7] K. Chemmangat, F. Ferranti, T. Dhaene, and L. Knockaert, "Tree-based sequential sampling algorithm for scalable macromodeling of highspeed systems," in IEEE 16th Workshop on Signal and Power Integrity (SPI), 2012, May 2012.

[8] B. Gustavsen and A. Semlyen, "Rational approximation of frequency domain responses by vector fitting," IEEE Trans. Power Delivery, vol. 14, no. 3, pp. 1052-1061, Jul. 1999.

[9] W. A. Weiser and S. E. Zarantonello, "A note on piecewise linear and multilinear table interpolation in many dimensions," Mathematics of Computation, vol. 50, no. 181, pp. 253-264, Jan. 1988.

[10] B. D. Anderson, S. Vongpanitlerd, Network Analysis and Synthesis. Englewood Cliffs, NJ: Prentice-Hall, 1973.

[11] S. Boyd, L. El Ghaoui, E. Feron and V. Balakrishnan, Linear Matrix Inequalities in System and Control Theory. Philadelphia, PA: SIAM, 1994, vol. 15. 